\title{
A model of cell motility leading to biphasic dependence of transport speed on adhesive strength
}

\author{
Yuan Lin \\ Department of Mechanical Engineering, The University of Hong Kong \\ Hong Kong SAR, China
}

\begin{abstract}
A rolling model for cell motility is proposed here where the movement of cell is treated as a result of the continuous release and growth of adhesions at the trailing and leading edge of the cell, respectively. The appearance of actin polymerization is key in this model as it breaks the symmetry of adhesion characteristics. The cell speed predicted here is in the correct range and exhibits a biphasic relationship with the cell-substrate adhesive strength which is consistent with experimental observations. We will show that this biphasic dependence of cell speed on adhesivity is due to the interplay between the energy dissipation associated with cell movement and the thermal fluctuations of actin filaments necessary for polymerization. Our results also suggest that the mobility of adhesion molecules is not only unnecessary but may actually limit cell motility.
\end{abstract}

Key words:

cell motility, adhesion and adhesives, chemo-mechanical processes 


\section{Introduction}

In response to certain stimuli, living cells are capable of changing shapes and moving on the extracellular matrix to which they adhere. This capability is essential to biological processes like wound healing where fibroblasts move around and repair the wounded region by laying down collagen fibers. Hence, from a therapeutic point of view, one way to expedite the healing process is to promote the motility of fibroblast. In other cases, such as in cancer treatment, it is desirable to limit the moving ability of cancer cells to minimize the possibility of metastasis. Although the phenomenon has been known for a long time, the nature of cell motility is still poorly understood. It is commonly believed that adhesion between the cell and the extracellular matrix, mediated by receptor-ligand binding interactions and serving to transmit the forces necessary for locomotion, plays a central role in the process. Various experiments have been conducted to investigate the influence of cell-substrate adhesive strength on cell speed, see Goodman et al. (1989) and Palecek et al. (1997) for example. A common feature of those experiment observations is the biphasic dependence of cell speed on adhesivity. Basically, a cell hardly moves when the adhesion between the substrate and the cell is either too strong or too weak. Whereas maximum cell motility is achieved when the adhesive strength is intermediate. In addition, numerous studies have convincingly demonstrated that cell motility is driven by actin polymerization (Preston et al., 1990; Theriot and Mitchison, 1991; Small, 1994). Usually, the continuous pushing on the membrane by polymerizing actin filaments leads to the formation of a flat protrusion region, often referred to as lamellipodium, extending from the main cell body in the 
direction of movement, as depicted in Figure 1. The exact force generation mechanism by actin polymerization is still under debate. Recently, several physical models have been proposed (Mogilner and Oster, 1996; Lin, 2009) which seem to be able to explain a variety of experimental observations on moving Listeria monocytogenes or beads driven purely by actin comet tails behind them (McGrath et al., 2003; Marcy et al., 2004).

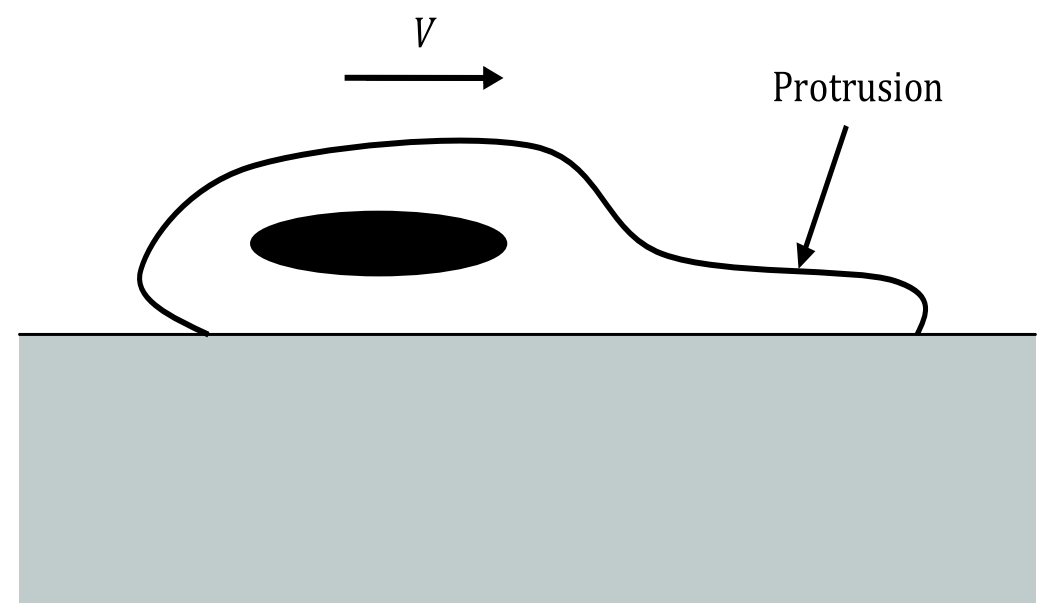

Figure 1: Schematic diagram of a motile cell.

Comprehensive reviews of the state of understanding of cell motility are available in Harris (1994) and Komnick et al. (1973). Theoretically, considerable progress has been made in trying to understand the basic mechanics and physics involved in cell locomotion. For example, a quantitative analysis on actin dynamics in moving cells has been conducted by Mogilner and Edelstein-Keshet (2002). By treating the cell as a viscoelastic solid, DiMilla et al. (1991) demonstrated the critical role of the transport of adhesion molecules, as well as the binding/unbinding reactions between them and their 
counterpart molecules, in cell locomotion. Their model indeed predicted that the cell migration speed reaches its maximum value at certain intermediate adhesive strength.

Despite these modeling efforts, we feel that several key components are still missing. First of all, to the best of our knowledge, the two most important factors in cell motility, namely the turnover of adhesions and the actin polymerization, have not been taken into account simultaneously by any existing model. For instance, actin polymerization is the sole focus in Mogilner and Edelstein-Keshet (2002), whereas it does not enter the formulation in DiMilla et al. (1991). To us, it is obvious that proper coordination between the turnover of adhesions and polymerization is absolutely essential for continuous cell movement, so any plausible model should address the interplay between them. Secondly, certain types of cells or amoebae, such as keratocyte and Hyalodiscus simplex, have long been found to advance by rolling (Harris, 1994; Anderson et al., 1996). During locomotion, a keratocyte (or Hyalodiscus simplex) does not show noticeable changes in its fan-like shape while maintaining a speed of about $0.15 \mu \mathrm{m} / \mathrm{s}$ (Komnick et al., 1973; Lee and Jacobson, 1997; Csucs et al., 2007). In comparison, migrating fibroblasts and many other slow-moving cells, with a typical speed of $\sim 60 \mu \mathrm{m} / \mathrm{h}$, undergo dramatic morphology changes and their movements are not smooth but jerky (Ware et al., 1998; Munevar et al., 2001). Despite some initial efforts (Anderson et al., 1996; Svitkina et al., 1997), a quantitative description on how cells move by rolling is still lacking. Aiming to address these issues, here we propose a simple model in which a motile cell is assumed to undergo a continuous rolling motion resulting from the release of cell-substrate adhesions 
at the rear end of the cell, as well as the growth of adhesions at the front end. As will be demonstrated later, actin polymerization plays an essential role in this model since it alters the adhesion characteristics at the leading and trailing edge of the cell by changing membrane tension and local peeling angles.

The rest of the paper is organized as follows. Detailed model formulation is given in the following section where descriptions of various processes involved in cell motility, such as actin polymerization, will be provided. After that, model predictions for two limiting cases where the diffusivity of adhesion molecules is either very large or very small are presented, and the implications for understanding various experimental observations are discussed. Finally, concluding remarks and thoughts are provided.

\section{Model Formulation}

Consider the situation in which a cell moves steadily from left to right at speed $V$, as shown in Figure 1. The bottom portion of the membrane surface adheres to the substrate while the top portion progresses at a speed of $2 \mathrm{~V}$, so the membrane surface appears to be rolling forward. In a frame of reference moving with the cell at speed $V$, the motion of the membrane resembles a conveyor belt with the top moving forward at speed $V$ and the bottom moving in the opposite direction at the same speed. As mentioned earlier, this type of locomotion has been observed in keratocytes (Anderson et al., 1996) and Hyalodiscus simplex (Harris, 1994). To avoid the unnecessary difficulties caused by the complex cell geometry, as illustrated in Figure 1, here we focus our attention on a simple model system where a cell is in 
contact with two flat substrates separated by a fixed distance $H$, as depicted in Figure 2.

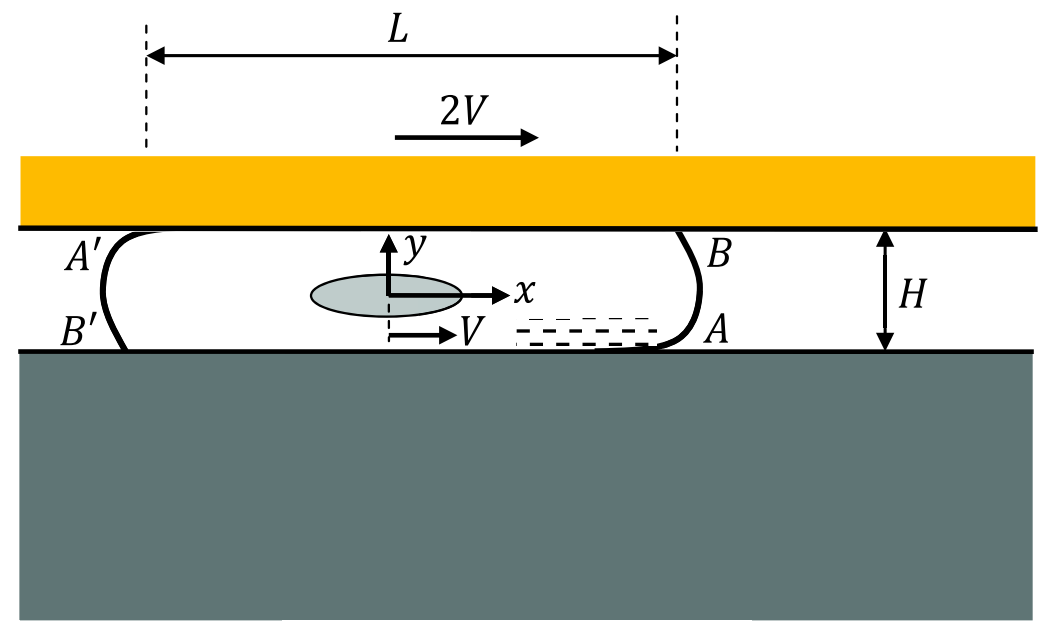

Stationary substrate

Figure 2: Diagram of the model system for studying cell motility.

The cell movement is assumed to be driven by the actin polymerization around two contact edges, denoted by $\mathrm{A}$ and $\mathrm{A}$ ' in Figure 2, between the cell and the substrate. To simplify the analysis, suppose that the bottom substrate remains stationary all the time while the top one can move freely in the horizontal direction. If no sliding between the membrane and substrate is allowed as along as they stick together, then the top substrate essentially moves at a speed twice as fast as the main body of the cell, see Figure 2.

\section{Transport of adhesion molecules}

Within the context of adhesive contact mechanics, a unique feature of cells is that adhesion is a result of specific bonding between receptors in the cell membrane and ligands on the substrate surface. Hence, the rolling motion 
considered here consists of formation of bonds at edge $\mathrm{A}$ and $\mathrm{A}^{\prime}$ in Figure 2, as well as the breaking of bonds at the other two edges, that is, edge B and B'. In addition to be able to bind to the substrate, free receptors also have the ability to diffuse within the membrane which has been demonstrated to be essential to the formation and evolution of the so-called tight cell adhesion regions (Freund and Lin, 2004; Boulbitch et al., 2001). Due to the obvious symmetry, only half of the whole cell membrane is examined here. In the reference frame moving with the main body of the cell at speed $V$, the local conservation of receptors inside the adhesion zone, i.e., segment B'A, requires

$$
\frac{\partial \xi}{\partial t}=D \frac{\partial^{2} \xi}{\partial x^{2}}+V \frac{\partial \xi}{\partial x}-k_{+}(x) \xi+k_{-}(x) \eta
$$

and

$$
\frac{\partial \eta}{\partial t}=V \frac{\partial \eta}{\partial x}+k_{+}(x) \xi-k_{-}(x) \eta
$$

where $\xi$ and $\eta$ are the densities of free and bounded receptor molecules, respectively. $D$ is the diffusion coefficient of receptors, $k_{+}$is the binding rate, or the so-called association rate, and $k_{-}$is the dissociation rate. $k_{+}$ and $k_{-}$, in their most general form, are functions of spatial coordinate $x$, as indicated in (1-2). However, to derive the essential results in the simplest way, here these rates are assumed to be constants within the adhesion region. Furthermore, if the energy reduction by the completion of forming a single bond is $C_{b} k T$, with $k T$ being the thermal energy, then these two constant rates can be expressed as

$$
k_{+}(x)=k_{0}, \quad k_{-}(x)=k_{0} e^{-C_{b}}
$$


where $k_{0}$ is a constant rate that has the physical dimension of $1 / \mathrm{s}$. We must point out that, in writing down (1-2), the ligand density on the substrate is assumed to be much higher than that of the receptor in the membrane and hence does not enter the formulation. On the other hand, in segment AB, where the membrane remains separated from the substrate, all receptors are free as illustrated in Figure 3 and, consequently, the conservation condition reduces to

$$
\frac{\partial \xi}{\partial t}=D \frac{\partial^{2} \xi}{\partial x^{2}}+V \frac{\partial \xi}{\partial x}
$$

We expect that there exists a thin processing zone near each adhesion edge, through which the receptor-ligand bond density decreases from a certain value just inside the adhesion region to zero outside it, as depicted in Figure 3. Discussions on what is possibly happening in this zone will be provided later. Similar to the bounded molecules, a jump in the free receptor concentration across this thin region is also admissible here, see Figure 3.

\section{Membrane tension and contact angles}

From a mechanics point of view, two key quantities, namely the membrane tension and the contact angle, determine the peeling or forming of adhesions between the cell and the substrate. Let the pressure difference between the inside and the outside of the cell be a constant, denoted as $p_{0}$. Suppose that actin polymerization is confined within a thin region, with thickness $d(d<<H)$, around the adhesion edge $\mathrm{A}$ as shown in Figure 4(a). In addition, assume that the effect of actin polymerization can be represented by an increase in local pressure from $p_{0}$ to $p$, see Figure 4(a). Neglecting shear stresses in the membrane, force equilibrium of segment $\mathrm{AB}$ requires 


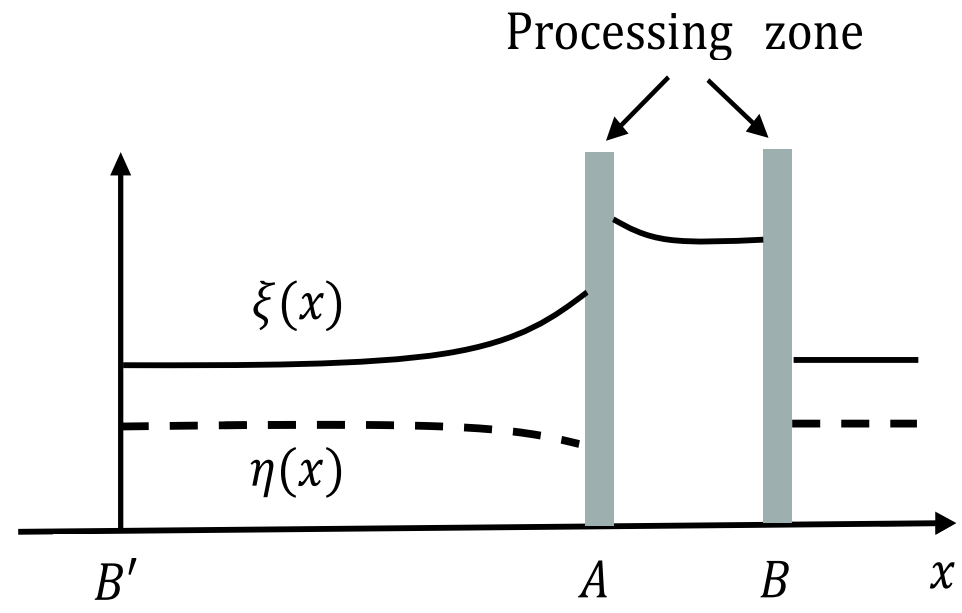

Figure 3: Schematic plot of the receptor density distribution within the cell membrane. The solid line represents the free receptors, whereas the dashed line corresponds to the bounded ones.

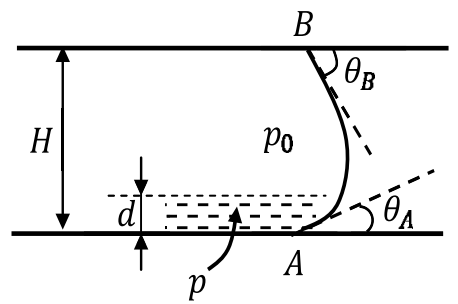

(a)

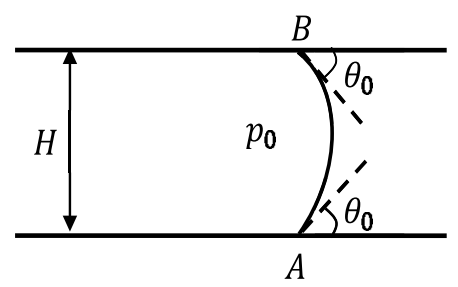

(b)

Figure 4: Illustration of the shape of the cell membrane. (a) - for a motile cell; (b) - for a stationary cell.

$$
n\left(\cos \theta_{A}+\cos \theta_{B}\right)-F=p_{0} H
$$

and 


$$
n \cos \theta_{B} H=\frac{p_{0} H^{2}}{2}
$$

where $F=p d$ is the net propelling force generated by actin polymerization, $n$ is the membrane tension, $\theta_{A}$ and $\theta_{B}$ are contact angles at adhesion edge $\mathrm{A}$ and $\mathrm{B}$, respectively. If there is no polymerization, i.e., $F=0$, these two angles are identical due to symmetry, as shown in Figure 4(b), and the cell remains stationary. Now it is clear that the appearance of actin polymerization breaks the symmetry and, consequently, causes the adhesion to grow and to be released at edge A and B respectively.

\section{Movement of adhesion front}

As mentioned earlier, adhesion between the cell and the substrate is due to the receptor-ligand bonds formed between them. Hence, the release or growth of adhesions must be accompanied by the breaking or forming of these molecular bonds. We proceed by assuming that the ligand molecules are immobilized on the substrate and form a rectangular pattern, as depicted in Figure 5. These ligand molecules, with spacing $\Delta$ among them, effectively serve as possible binding sites for a cell to adhere to the substrate.

To balance the peeling force induced by membrane tension, bonds near the adhesion edge are necessarily to be stretched. Following the same argument as in Lin and Freund (2007) and Boulbitch (2003), we assume the bending rigidity of the membrane itself is small and, consequently, only a single layer (with width $\Delta$ ) of bonds nearest to the edge is stretched, see Figure 5. If we put the origin of the reference frame at the adhesion front, as shown in Figure 5, then the normal force $q$ supported by a single bond within this stretched region is 


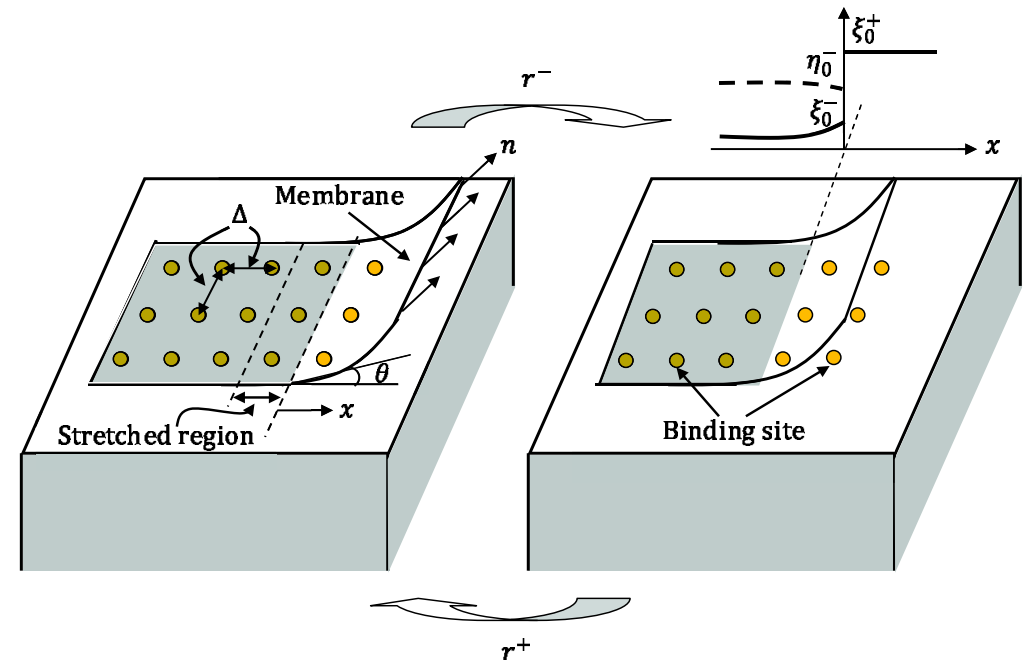

Figure 5: Illustration of the movement of an adhesion front. The shaded area represents the portion of membrane that adheres to the substrate.

$$
q=\frac{n \sin \theta}{\eta_{0}^{-} \Delta}
$$

where $\eta_{0}^{-}$is the macroscopic receptor-ligand bond concentration just inside the adhesion zone, $\theta$ is the contact angle and $n$ is the membrane tension. It is natural to believe that the appearance of a stretching force makes the breaking of bonds easier. This issue was first examined by Bell (1978) who proposed that the dissociation rate of a bond increases exponentially with the force acting on it. In light of (3), the breaking rate $k_{-}(q)$ for bonds in the stretched region now becomes

$$
k_{-}(q)=k_{0} e^{-C_{b}} e^{q a / k T}=k_{0} e^{-C_{b}} e^{q / f_{0}} .
$$

In which $f_{0}=k T / a$, and $a \sim 0.1 \mathrm{~nm}$ is a constant length which has the physical meaning of the approximate width of the potential well representing 
the actual bond. We must point out that (8) implies only the normal force $q$ affects the breaking of bonds. However, in reality, depending on the actual structure as well as the dissociation pathway of the bond concerned, the tangential force acting on it may also play a role in its enforced breaking, a possibility that has been neglected here for simplicity. From (8), the rate for breaking all the bonds inside the stretched region can be expressed as

$$
r^{-}=k_{0}\left[e^{-C_{b}} e^{q / f_{0}}-\frac{\xi_{0}^{-}}{\eta_{0}^{-}}\right]
$$

where, similar to $\eta_{0}^{-}, \xi_{0}^{-}$is the macroscopic free receptor concentration just inside the adhesion region. Hence, at any instance, a single layer of adhesions can be released with a rate $r^{-}$given in (9). On the other hand, we expect a new layer of adhesions, with the same width $\Delta$, can be formed at a different rate $r^{+}$, as shown in Figure 5. To complete such a transition, the bond concentration must grow from zero, in the original configuration where the layer separates from the substrate, to $\eta_{0}^{-}$when the layer becomes attached to the substrate. Hence, the transition rate $r^{+}$can be estimated as

$$
r^{+}=k_{0} \frac{\xi_{0}^{+}}{\eta_{0}^{-}}
$$

where it is assumed that the free receptors just outside the adhesion zone, with concentration $\xi_{0}^{+}$, have the same association rate $k_{0}$ as those inside. Similar to Lin and Freund (2008), here the movement of adhesion front is treated as a stochastic process where the edge can move forward and backward with transition rates $r^{+}$and $r^{-}$respectively. Hence, the macroscopic velocity of the adhesion front is simply 


$$
V=\left(r^{+}-r^{-}\right) \Delta
$$

An immediate consequence of (11) is that there exists a maximum speed $V_{\max }$ for the growth of adhesions, which is achieved when the peeling force acting on the adhesion edge vanishes, that is

$$
V_{\text {max }}=k_{0} \Delta\left[\frac{\xi_{0}^{+}}{\eta_{0}^{-}}+\frac{\xi_{0}^{-}}{\eta_{0}^{-}}-e^{-C_{b}}\right]
$$

Force generation by polymerization

So far we have not touched a key issue in cell motility, namely the force generation by polymerization. As mentioned earlier, the pushing on the cell membrane by actin filaments breaks the symmetry of adhesion characteristics and, consequently, causes the cell to advance by rolling. In the discussions above, it is simply assumed that a net propelling force $F$ is generated by polymerization without any elaboration on the actual mechanism behind it.

As depicted in Figure 6, polymerizing filaments grow by adding actin monomers to their barbed ends which, in return, keep pushing the cell membrane. However, a question naturally arises from this picture is that how can monomers be added to the barbed ends which are already in contact with the membrane? A plausible explanation was proposed by Mogilner and Oster $(1996,2003)$ where the central idea is that thermal excitations are large enough to bend the actin filament and create a gap $\delta$ between the filament tip and the load surface which makes the addition of monomers possible, as illustrated in Figure 7. Recently, on the basis of this idea, a generalized formulation has been proposed which seems to be capable of explaining various experimental observations (Lin, 2009). A common finding of the studies 


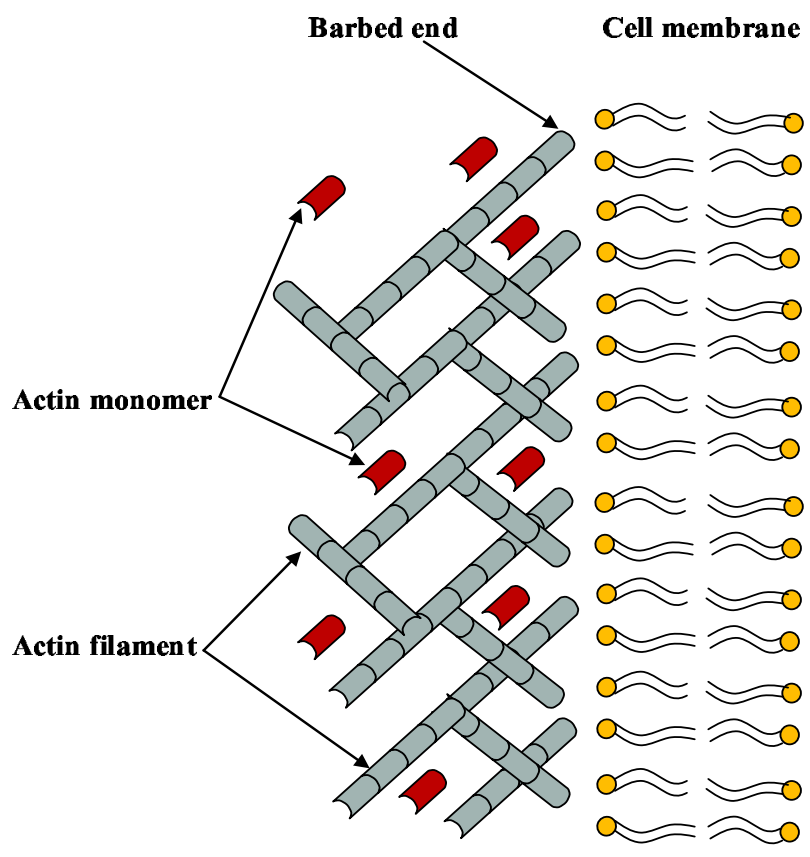

Figure 6: Schematic plot of the polymerizing actin filaments near the cell membrane.

mentioned above is that the magnitude of the propelling force $f$ generated by a single polymerizing filament decreases rapidly with the moving speed $V$ of the load surface. Particularly, in the regime where the propelling force is large and depolymerization is negligible, the force-velocity relationship takes the asymptotic form (Mogilner and Oster, 1996, 2003)

$$
\frac{V}{V_{p o l}}=e^{-f / f_{p}}
$$

with

$$
f_{p}=k T / \delta_{0}
$$

where $\delta_{0}$ is the projected actin monomer size. $V_{\text {pol }}$ has the physical meaning 
of the maximum speed at which the filament can grow, i.e., the free polymerization speed. It is easy to understand that this speed must be limited by how fast the adhesion can grow, that is, it can not exceed $V_{\max }$ as defined in (12). Here, for simplicity, $V_{\text {pol }}$ is chosen as

$$
V_{p o l}=V_{\max }
$$

A simple explanation for the exponential decay of $V$ corresponding to $f$, as predicted by (13), is that, to generate large propelling forces, the filaments are necessarily to be highly bended which makes it increasingly difficult for tips to fluctuate away from the load surface. As a result, chances for adding monomers to filament tips become slim. Obviously, here the growing speed that the polymerizing filaments can achieve is limited by the fluctuating ability of tips. For this reason, we refer to this regime as the fluctuation dominant regime.

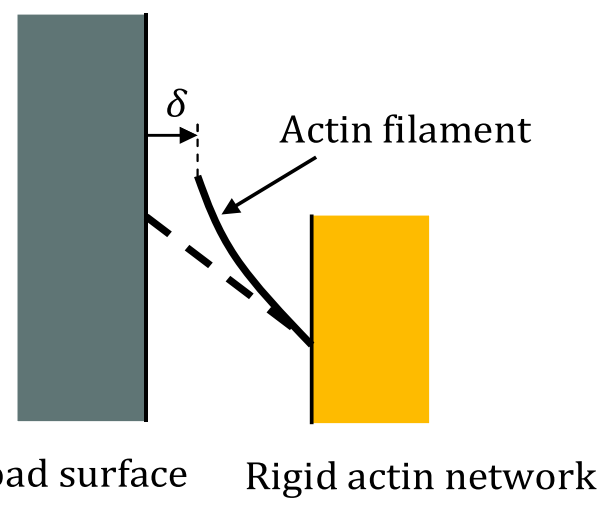

Figure 7: Configurations of actin filament. The dashed line represents the undeformed filament and the solid line corresponds to the deformed one due to thermal excitations. 
On the other hand, it can easily be verified that, for the configuration shown in Figure 2, the rate of work done by actin polymerization is

$$
\dot{W}_{p}=2 F V
$$

From the power balance point of view, this quantity must exceed the rate of energy dissipation $\dot{\mathcal{D}}$ associated with cell movement, that is

$$
\dot{W}_{p} \geq \dot{\mathcal{D}}
$$

The energy dissipation may come from, for example, the viscous nature of the whole cell body or the diffusion of adhesion molecules along cell membrane (Freund and Lin, 2004; Gao et al., 2005). To simplify the analysis, we only consider the viscous dissipation here, which is expected to be the dominant source when the length of a cell is much larger than its height, i.e., $L>>H$ in Figure 2. If simple shear flow is assumed, then the rate of viscous dissipation inside the cell is

$$
\dot{\mathcal{D}} \approx 2 \mu L \frac{V^{2}}{H}
$$

in which $\mu$ is the viscosity of the fluid inside the cell. From (16-18), it is clear that the propelling force $f$ generated by a single filament and the cell speed $V$ must satisfy the following inequality

$$
f \geq \frac{\mu L \lambda^{2}}{d H} V=\alpha V
$$

where $\lambda$ is the average spacing between polymerizing filaments at the leading edge of the motile cell. (19) shows that when $f$ is small the cell speed $V$ must 
be small as well because otherwise the power generated by polymerization will not be able to overcome the energy dissipation. Hence, we refer to the regime where $f$ is small as the dissipation dominant regime.

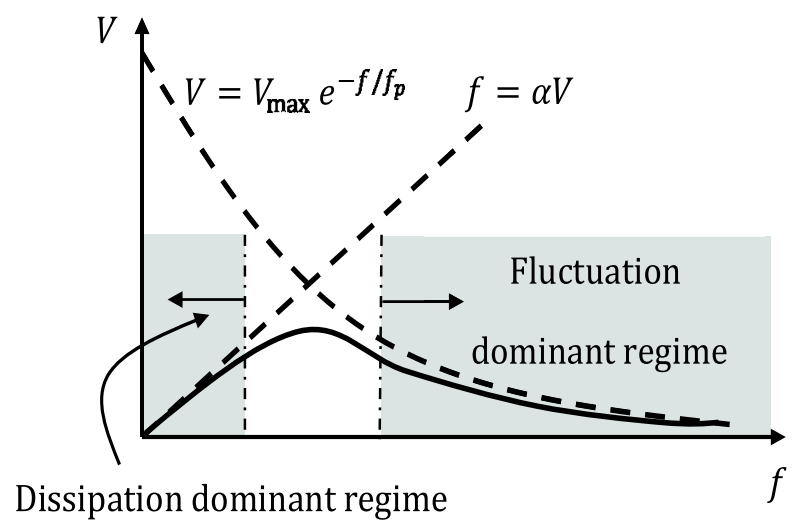

Figure 8: Illustration of the force-velocity relationship of polymerizing filaments.

The above discussions are summarized in Figure 8. Basically, we expect the actual force-velocity relationship for polymerizing filaments to follow (13) when the propelling force is very large, and to approach the prediction by (19) when $f$ is very small. However, it is unlikely that a closed form relationship, valid for arbitrary propelling force values, can be obtained. For simplicity, here $V$ as a function of $f$ is assumed to take the form

$$
V=\min \left[e^{-f / f_{p}} V_{\max }, \frac{f}{\alpha}\right]
$$

\section{Results and Discussions}

To demonstrate the main features of the model presented here, we proceed by considering two limiting cases where the diffusivity of receptors is either 
very low or very high because, as will be shown later, solutions can be found in a rather simple manner under these limiting conditions .

Low diffusivity case: $D \rightarrow 0$

In this case, there is no diffusion of receptors along the membrane. As a result, the local receptor concentration remains as a constant, that is

$$
\xi(x)+\eta(x)=\xi_{0}
$$

where $\xi_{0}$ is the initial receptor concentration in the membrane before the cell is brought into contact with the substrates. Under such circumstance, a simple steady-state solution to (1) and (2) is given by

$$
\xi(x)=\xi_{e q}=\frac{1}{1+e^{C b}} \xi_{0}, \quad \eta(x)=\eta_{e q}=\frac{e^{C b}}{1+e^{C b}} \xi_{0}
$$

whereas in the free portion of membrane, i.e., segment AB in Figure 3, the solution to (4) is simply

$$
\xi(x)=\xi_{0}
$$

So, for the leading edge of the cell, i.e., edge A in Figure 2, one has $\xi_{0}^{-}=\xi_{\text {eq }}$, $\eta_{0}^{-}=\eta_{e q}$ and $\xi_{0}^{+}=\xi_{0}$. We proceed by nondimensionalizing the problem as follows

$$
\begin{gathered}
\hat{\xi}=\xi \Delta^{2}, \quad \hat{\eta}=\eta \Delta^{2}, \quad \hat{F}=\frac{F}{p_{0} H}, \quad \hat{n}=\frac{n}{p_{0} H}, \quad \hat{V}=\frac{V}{k_{0} \Delta} \\
\hat{f}_{0}=\frac{k T}{p_{0} H a \Delta}, \quad \hat{f}_{p}=\frac{k T d}{p_{0} H \lambda^{2} \delta_{0}}, \quad \hat{\alpha}=\frac{\mu L k_{0} \Delta}{p_{0} H^{2}}
\end{gathered}
$$


In light of (7-11), the normalized speed of edge A takes the form

$$
\hat{V}_{A}=\hat{V}=\frac{\hat{\xi}_{0}}{\hat{\eta}_{e q}}+\frac{\hat{\xi}_{e q}}{\hat{\eta}_{e q}}-e^{-C_{b}} e^{\hat{n} \sin \theta_{A} / \hat{\eta}_{e q} \hat{f}_{0}}
$$

Similarly, for edge B in Figure 2, we have

$$
\hat{V}_{B}=-\hat{V}=\frac{\hat{\xi}_{0}}{\hat{\eta}_{e q}}+\frac{\hat{\xi}_{e q}}{\hat{\eta}_{e q}}-e^{-C_{b}} e^{\hat{n} \sin \theta_{B} / \hat{\eta}_{e q} \hat{f}_{0}}
$$

In this case, the maximum speed at which the adhesion can grow is

$$
\hat{V}_{\max }=1+e^{-C_{b}}
$$

The force balance equations (5-6) become

$$
\hat{n}\left(\cos \theta_{A}+\cos \theta_{B}\right)-\hat{F}=1
$$

and

$$
\hat{n} \cos \theta_{B}=\frac{1}{2}
$$

Finally, the force-velocity relationship (20), summarizing the mechanics of polymerization, takes the normalized form

$$
\hat{V}=\min \left[e^{-\hat{F} / \hat{f}_{p}} \hat{V}_{\max }, \frac{\hat{F}}{\hat{\alpha}}\right]
$$

Equations (25-26) and (28-30) form a mathematically closed system from which five unknown variables, that is, $\hat{n}, \theta_{A}, \theta_{B}, \hat{F}$, and $\hat{V}$, can be solved. Before presenting any results, the values of a set of parameters must be chosen first. The projected monomer size $\delta_{0}$ of actin is about $2.2 \mathrm{~nm}$ (Pollard, 1986; Lin, 2009). We estimate the length of a cell, denoted as $L$ in Figure 2, to 
be around $30 \mu \mathrm{m}$ for a keratocyte or Hyalodiscus simplex (Harris, 1994; Lee and Jacobson, 1997). The height of the cell is assumed to be in the range of 0.5 to $1 \mu \mathrm{m}$, whereas the thickness of the polymerization region is taken to be around 50-100 nm. Since the height of the cell is small, we expect that organelles and cytoskeleton filaments occupy most of the cell volume. Hence, the viscosity of the cell body is estimated to be around $20 \mathrm{P}$ here, which is close to the viscosity of the cytoplasm of the cell (Dembo, 1989). The pressure $p_{0}$ varies across cell types. Here, $p_{o}$ is assumed to be around $100 \mathrm{~Pa}$, which is a typical value for Walker carcinosarcoma cells (Rentsch and Keller, 2000).

The diameter of an integrin molecule is reported to be around $12 \mathrm{~nm}$ (Hynes, 1992), experiment observations also suggest that the adhesion becomes unstable when the spacing between bonds exceeds $\sim 80 \mathrm{~nm}$ (Arnold et al., 2004). Hence, the value of $\Delta$ is estimated to be around $50 \mathrm{~nm}$ here. The time required for binding between receptor and ligand to happen is believed to be in the order of a second (Balaban et al., 2001), so here $k_{0}$ is estimated to be in the range of $1-10 \mathrm{~s}^{-1}$. As mentioned before, $a$ should be around 0.1 $\mathrm{nm}$, and it is reasonable to believe that the spacing between actin filaments is in the order of $50 \mathrm{~nm}$. The values of all parameters used here are gathered in Table 1. Taking $H=500 \mathrm{~nm}$ and $d=50 \mathrm{~nm}$, the three dimensionless parameters $\hat{f}_{0}, \hat{f}_{p}$ and $\hat{\alpha}$ are estimated to be in the order of 10,1 and 0.5 , respectively.

Choosing $\hat{f}_{0}=8, \hat{f}_{p}=1$ and $\hat{\alpha}=1$, the relationship between the cell speed and the binding energy is shown in Figure 9 for different initial receptor concentrations. The most striking feature shown in Figure 9 is that, for 
Table 1: Parameter values

\begin{tabular}{|c|c|c|}
\hline Notation & Meaning & Value \\
\hline$L$ & Length of the cell & $\sim 30 \mu \mathrm{m}$ \\
$H$ & Height of the cell & $0.5-1 \mu \mathrm{m}$ \\
$d$ & Thickness of the polymerization region & $50-100 \mathrm{~nm}$ \\
$\delta_{0}$ & Projected actin monomer size & $\sim 2.2 \mathrm{~nm}$ \\
$\Delta$ & Spacing between possible binding sites on the substrate & $\sim 50 \mathrm{~nm}$ \\
$\lambda$ & Average spacing between actin filaments & $\sim 50 \mathrm{~nm}$ \\
$k_{0}$ & Binding rate & $1-10 \mathrm{~s}^{-1}$ \\
$a$ & Approximate width of the potential well representing the bond & $\sim 0.1 \mathrm{~nm}$ \\
$\mu$ & Viscosity of the cell body & $\sim 20 \mathrm{P}$ \\
$p_{0}$ & Pressure inside the cell & $\sim 100 \mathrm{~Pa}$ \\
\hline
\end{tabular}

any given value of $\xi_{0}$, the maximum cell speed is achieved at a moderate binding energy. Whereas cell speed becomes small when the binding energy is either too high or too low. Notice that here $C_{b}$ approximately represents the cell-substrate adhesive strength. The biphasic relationship between the cell speed and the adhesivity has been reported from multiple experimental observations, see de Beus and Jacobson (1998) and Palecek et al. (1997) for example. In light of our model, this phenomenon can be explained as follows. If the adhesion between the cell and the substrate is weak, the propelling force $F$ generated by polymerizing filaments must be small because otherwise adhesions at the leading edge of a migrating cell will not be able to grow. Consequently, according to Figure 8, cells move slowly due to energy dissipation. On the other hand, to peel the membrane from the substrate at 
the trailing edge of a motile cell, $F$ is necessary to be large when the adhesion is strong. As illustrated in Figure 8, in this case the cell speed, or equivalently the growing velocity of actin filaments, will also be small because the large load makes it increasingly difficult for filament tips to fluctuate away from the cell membrane, which is essential for the growth of filaments.

The true cell speed can be obtained by multiplying the dimensionless value $\hat{V}$ with $k_{0} \Delta$. Choosing $k_{0} \Delta=200 \mathrm{~nm} / \mathrm{s}$, a typical value, Figure 9 suggests that cells move with a speed of $\sim 0.15 \mu \mathrm{m} / \mathrm{s}$ which is consistent with the experimental observations of keratocytes (Lee and Jacobson, 1997; Csucs et al., 2007). Another important feature shown in Figure 9 is that, for a given receptor density, there exists a critical value of the binding energy below which cells are not able to move. This minimum binding energy as a function of receptor concentration is shown in Figure 10. Clearly, this critical value decreases monotonically with increasing receptor density. It is interesting to point out that several studies (Lin et al., 2008; Wei, 2008) have demonstrated the existence of a critical adhesive strength below which adhesion between a cell and substrate becomes unstable due to steric repulsions. Of course, our formulation does not take into account any steric effect, which will impose additional constraint on the minimum binding energy necessary for cell locomotion.

Alternatively, we can examine how cells move differently under various receptor concentrations for a given binding energy. Figure 11 shows the cell speed as a function of receptor density for three binding energy values, i.e., $C_{b}=2,5$ and 10. Again, the cell speed predicted here exhibits a biphasic relationship with the cell-substrate adhesive strength. Figure 11 also suggests 


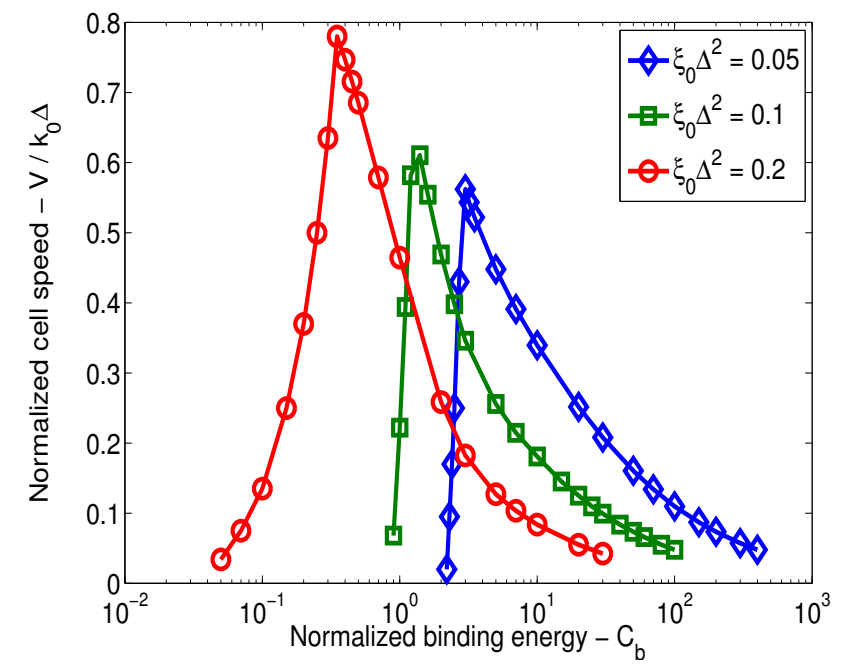

Figure 9: Relationship between the cell speed and the binding energy.

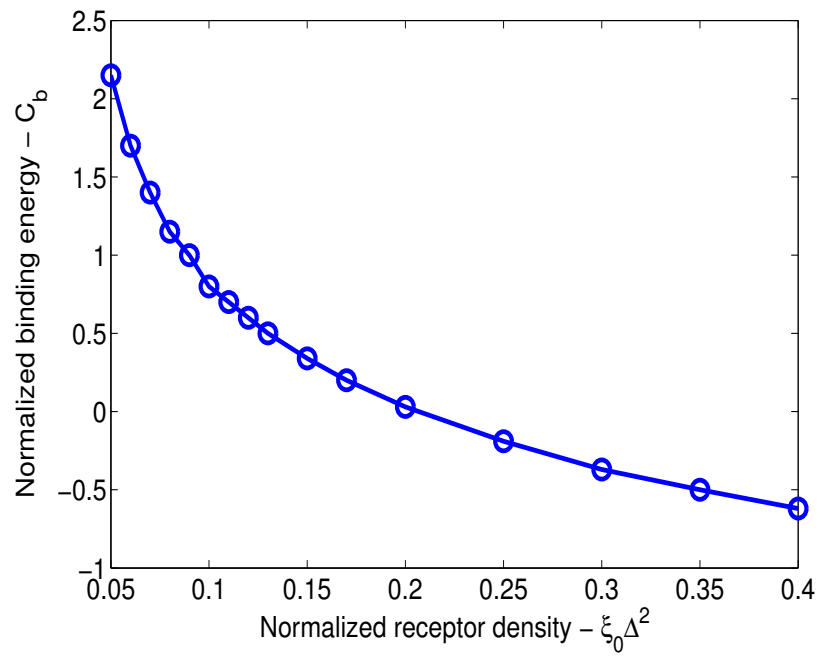

Figure 10: The minimum binding energy necessary for cell locomotion as a function of receptor concentration.

that the maximum speed a cell can achieve is insensitive to the binding energy, but the peak position shifts to lower receptor concentrations with 
increasing $C_{b}$. It is interesting to point out that similar trends have been observed for CHO cells. Specifically, Palecek et al. (1997) found that varying the integrin-ligand affinity, or equivalently the binding energy, by transfecting CHO cells with certain chemical agents does not affect the maximum cell speed, but instead leads to the shift of peak position. Note that the energy reduction by forming a single receptor-ligand bond is believed to be in the range of 5-25 kT (Boal, 2002), which seems to suggest that the findings here may indeed be relevant to actual cell migration. However, we must also point out that one needs to be very cautious in comparing the model predictions here to the aforementioned experiments because the typical speed of $\mathrm{CHO}$ cells is around $10 \mu \mathrm{m} / \mathrm{h}$ (Palecek et al., 1997) which is at least one magnitude lower than that of keratocyte or Hyalodiscus simplex, and hence it is unlikely that $\mathrm{CHO}$ cells advance by rolling. Of course, we would like to compare our model to similar experimental results obtained on keratocyte or Hyalodiscus simplex, unfortunately, despite extensive efforts, we were unable to find such data in the literature.

High diffusivity case: $D \rightarrow \infty$

As $D \rightarrow \infty$, the free receptors must distribute uniformly in the membrane. Assuming $L>>H$ in Figure 2, the receptor distributions in the adhesion region, i.e., segment B'A in Figure 3, are identical to those shown in (22). However, in the free portion of membrane, i.e., segment AB in Figure 3, the receptor distribution becomes

$$
\xi(x)=\xi_{e q}=\frac{1}{1+e^{C b}} \xi_{0}
$$

Consequently, the moving speeds of two edges now take the form 


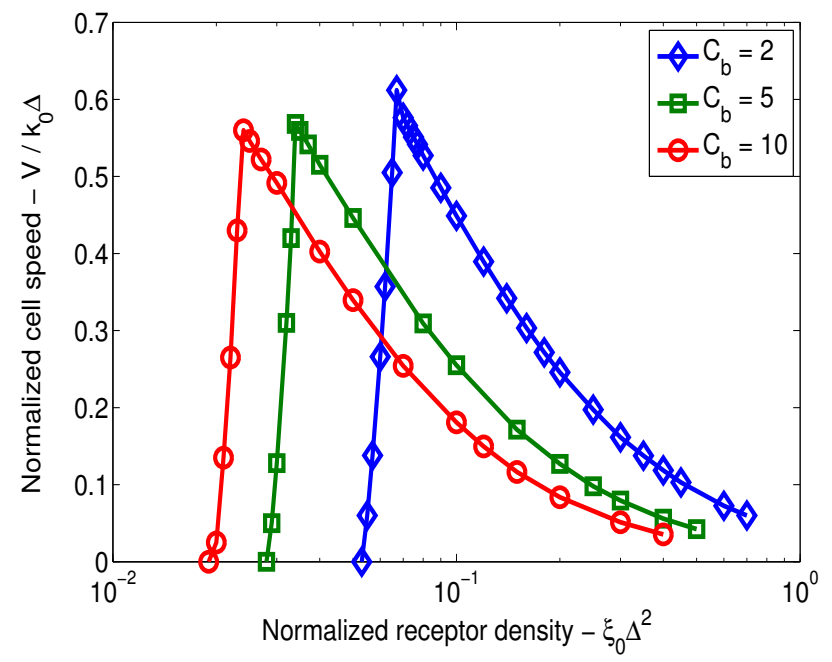

Figure 11: The cell speed as a function of the receptor concentration.

$$
\hat{V}_{A}=\hat{V}=2 \frac{\hat{\xi}_{e q}}{\hat{\eta}_{e q}}-e^{-C_{b}} e^{\hat{n} \sin \theta_{A} / \hat{\eta}_{e q} \hat{f}_{0}}
$$

and

$$
\hat{V}_{B}=-\hat{V}=2 \frac{\hat{\xi}_{e q}}{\hat{\eta}_{e q}}-e^{-C_{b}} e^{\hat{n} \sin \theta_{B} / \hat{\eta}_{e q} \hat{f}_{0}}
$$

Under such circumstance, the maximum speed at which the adhesion can grow becomes

$$
\hat{V}_{\max }=e^{-C_{b}}
$$

Choosing the same parameters as in Figure 9, the cell speed as a function of binding energy for $\xi_{0} \Delta^{2}=0.2$ is shown in Figure 12. In comparison, the cell speed corresponding to $D=0$ is also given in Figure 12. Clearly, our model predicts that the cell speed will be reduced by the increase in 
receptor mobility. This seems to be counterintuitive since one would expect that higher diffusivity allows receptors to move from the rear end to the front end of the cell faster and consequently enhances cell motility. However, in the rolling model presented here, the motion of membrane itself can bring free receptors to the front edge of the cell and hence mobility of receptors is no longer indispensable. Furthermore, the free receptor concentrations in the free portion of membrane will be diluted as D increases, see (23) and (31), which eventually leads to the decrease in the maximum growth speed of adhesions, i.e., $V_{\max }$, as shown in (27) and (34).

At this point, it is informative to consider whether diffusion of adhesion molecules is important in actual cell locomotion. The diffusivity of receptors has been found to be in the range of $10^{-12}-10^{-9} \mathrm{~cm}^{2} / \mathrm{s}$ (Jacobson et al., 1987; Duband et al., 1988), so the dimensionless parameter $\hat{D}=D / k_{0} \Delta^{2}$ is estimated to vary from 0.01 to 10 . This suggests that, depending on the specific type of receptors, the diffusion of adhesion molecules can change from negligible $(\hat{D} \sim 0.01)$ to rather substantial $(\hat{D} \sim 10)$. For intermediate $\hat{D}$ values, the receptor distributions have to be solved according to (1), (2) and (4) with the help of certain auxiliary conditions, such as the global conservation of receptors, but this issue is not pursued here.

As mentioned before, a quantitative comparison between the model and experiment is not available at present due to the lack of relevant experimental data. Nevertheless, it is hoped that our results can provide guidance for the design of future experiments. For example, in the rolling model presented here, receptor mobility is predicted to have a negative effect on the moving capability of cells; our analysis also suggests that the maximum cell speed is 


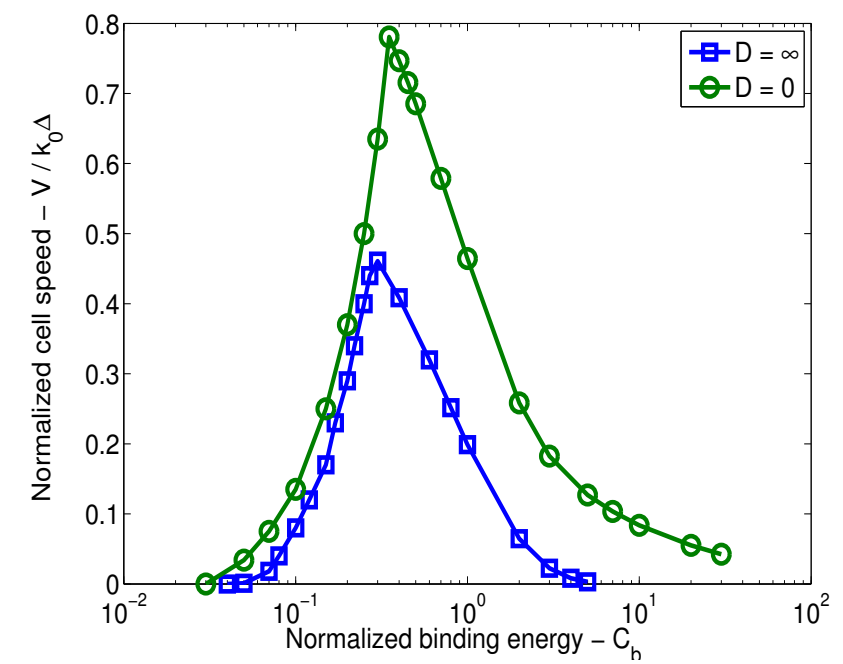

Figure 12: Relationship between the cell speed and the binding energy.

insensitive to the binding energy when the diffusivity of receptors is negligible. These predictions can be specifically tested in the future by carefully designed experiment, and ,despite the outcome, such interplay between theory and experiment will undoubtedly give us a better understanding of cell locomotion.

\section{Concluding Remarks}

In this paper, a model for cell motility is proposed based on the observation that actin polymerization breaks the symmetry of adhesion characteristics at the leading and trailing edge of the cell. As a result, the cell tends to advance by the continuous release and growth of adhesions at the rear and front end of the cell respectively. We believe that two important goals have been achieved in this work. First, to the best of our knowledge, our work is the first attempt to provide a quantitative description of the movement 
of cells like keratocyte and Hyalodiscus simplex, which have been known to advance by rolling. Secondly, the model presented here has simultaneously taken into account the two most important factors in cell motility, namely the force generation by actin polymerization and the turnover of adhesions, which are often examined separately in previous studies. By choosing reasonable parameter values, the cell speed predicted here is in the correct range $(\sim 0.15 \mu \mathrm{m} / \mathrm{s})$ and exhibits a biphasic relationship with the cell-substrate adhesive strength which is consistent with experiment observations. Our model also provides a physical explanation for this biphasic behavior of cell speed with adhesivity. Basically, when the adhesion is weak the propelling force generated by actin polymerization must be small, and the cell moves slowly due to energy dissipation. On the other hand, the force generated by actin filaments is expected to be large when adhesion is strong, and the cell speed in this case is also small because the possibility for filaments to fluctuate away from the membrane, which is essential for polymerization to take place, has been greatly reduced. Predictions here may be used in the design of future experiments and further increase our understanding on how cells actually move by rolling.

A number of assumptions have been incorporated in the development of the model which render the formulation mathematically tractable and the essential features of the model relatively transparent. As a result of these simplifications, several important features of the process of cell motion have been overlooked which certainly deserve further investigation. To begin with, the adhesion between the cell and substrate is assumed to be uniform here. However, numerous experiments have convincingly demonstrated that 
this is hardly the case, instead discrete micron-size tight contact sites, often referred to as focal adhesions, will be formed between the cell and substrate (Lee and Jacobson, 1997; Balaban et al., 2001; Zaidel-Bar et al., 2004). The formation of focal adhesions is believed to involve the assembly of different proteins, including vinculin and paxillin (Zamir and Geiger, 2001), and to be influenced by the tractions acting on them through the so-called stress fibers, which are actin filaments and their associated myosin based molecular motors (Balaban et al., 2001; Tan et al., 2003; Novak et al., 2004; Deshpande et al., 2008). Intensive efforts have been made to investigate the possible correlation between tractions transmitted through individual focal adhesions and cell movement (Dembo and Wang, 1999; Beningo et al., 2001). Clearly, this important issue has not been taken into account in our model. On top of that, we actually only considered the force-velocity relationship of polymerizing filaments in a motile cell under two extreme conditions where the force is either very large or very small. Obviously, this description is incomplete and a more rigorous analysis, similar to those in Mogilner and Oster (1996) and Lin (2009), should be conducted in the future to address this problem. Last but not least, the geometry of the cell in our model system, as shown in Figure 2, is very simple. In reality, a motile cell takes on a much more complicated shape, see Anderson et al. (1996) and Svitkina et al. (1997) for example, and hence the pattern of membrane flow may not be quite as simple as the movement of a conveyor belt as adopted here. This issue, like others mentioned above, is left for future studies.

Despite the aforementioned simplifications, we believe that the model does capture a number of important features of cell motility, and the con- 
clusion that cell speed exhibits a biphasic dependence on adhesivity due to the interplay between the energy dissipation associated with cell movement and the thermal fluctuations of actin filaments, necessary for polymerization, should be rather robust. In addition, we feel that the the formulation presented here may serve as a framework for further studies on cell locomotion where more realistic features can be added.

\section{Acknowledgment}

This investigation was supported by the Seed Funding Programme for Basic Research from The University of Hong Kong (Project No. 200809159003).

\section{References}

- Anderson, K.I., Wang, Y.-L., and Small, J.V., 1996. Coordination of protrusion and translocation of the keratocyte involves rolling of the cell body. The Journal of Cell Biology 134, 1209-1218.

- Arnold, M., Cavalcanti-Adam, E.A., Glass, R., Blümmel, J., Eck, W., Kantlehner, M., Kessler, H., and Spatz, J.P., 2004. Activation of integrin function by nanopatterned adhesive interfaces. ChemPhysChem 5, 383-388.

- Balaban, N.Q., Schwarz, U.S., Riveline, D., Goichberg, P., Tzur, G., Sabanay, I., Mahalu, D., Safran, S., Bershadsky, A., Addadi, L., and Geiger, B., 2001. Force and focal adhesion assembly: a close relationship studied using elastic micropatterned substrates. Nature Cell Biology 3, 466-472. 
- Bell, G.I., 1978. Models for the specific adhesion of cells to cells. Science 200, 618-627.

- Beningo, K.A., Dembo, M., Kaverina, I., Small, J.V., and Wang, Y.L., 2001. Nascent focal adhesions are responsible for the generation of strong propulsive forces in migrating fibroblasts. The Journal of Cell Biology 153, 881-887.

- Boal, D., 2002. Mechanics of the cell. Cambridge University Press, Cambridge.

- Boulbitch, A., 2003. Enforced unbinding of biomembranes whose mutual adhesion is meditated by a specific interaction. European Biophysics Journal 31, 637-642.

- Boulbitch, A., Guttenberg, Z., and Sackmann, E., 2001. Kinetics of membrane adhesion mediated by ligand-receptor interaction studied with a biomimetic system. Biophysical Journal 81, 2743-2751.

- Csucs, G., Quirin, K., and Danuser, G., 2007. Cell motility and the cytoskeleton 64, 856-867.

- de Beus, E., and Jacobson, K., 1998. Integrin involvement in keratocyte locomotion. Cell Motility and the Cytoskeleton 41, 126-137.

- Dembo, M., 1989. Mechanics and control of the cytoskeleton in Amoeba proteus. Biophysical Journal 55, 1053-1080.

- Dembo, M., and Wang, Y.-L., 1999. Stresses at the cell-to-substrate interface during locomotion of fibroblasts. Biophysical Journal 76, 23072316.

- Deshpande, V.S., Mrksich, M., McMeeking, R.M., and Evans, A.G., 2008. A bio-mechanical model for coupling cell contractility with focal 
adhesion formation. Journal of the Mechanics and Physics of Solids 56, 1484-1510.

- DiMilla, P.A., Barbee, K., and Lauffenburger, D.A., 1991. Mathematical model for the effects of adhesion and mechanics on cell migration speed. Biophysical Journal 60, 15-37.

- Duband, J.-L., Nuckolls, G.H., Ishihara, A., Hasegawa, T., Yamada, K.M., Thiery, J.P., and Jacobson, K., 1988. Fibronectin receptor exhibits high lateral mobility in embryonic locomoting cells but is immobile in focal contacts and fibrillar streaks in stationary cells. The Journal of Cell Biology 107, 1385-1396.

- Freund, L.B., and Lin, Y., 2004. The role of binder mobility in spontaneous adhesive contact and implications for cell adhesion. Journal of the Mechanics and Physics of Solids 52, 2455-2472.

- Gao, H., Shi, W., and Freund, L.B., 2005. Mechanics of receptormediated endocytosis. Proceedings of the National Academy of Sciences 102, 9469-9474.

- Goodman, S.L., Risse, G., and von der Mark, K., 1989. The E8 subfragment of laminin promotes locomotion of myoblasts over extracellular matrix. The Journal of Cell Biology 109, 799-809.

- Harris, A.K., 1994. Locomotion of tissue culture cells considered in relation to ameboid locomotion. International Review of Cytology 150, $35-67$.

- Hynes, R.O., 1992. Integrins: versatility, modulation, and signaling in cell adhesion. Cell 69, 11-25.

- Jacobson, K., Ishihara, A., and Inman, R., 1987. Lateral diffusion of 
proteins in membranes. Annual Review of Physiology 49, 163-175.

- Komnick, H., Stockem, W., and Wohlfarth-Bottermann, K.E., 1973. Cell motility: mechanicnism in protoplasmic streaming and ameboid movement. International Review of Cytology 34, 169-249.

- Lee, J., and Jacobson, K., 1997. The composition and dynamics of cell-substratum adhesion in locomoting fish keratocytes. Journal of Cell Science 110, 2833-2844.

- Lin, Y., 2009. Mechanics model for actin-based motility. Physical Review E 79, 021916.

- Lin, Y., and Freund, L.B., 2007. Forced detachment of a vesicle in adhesive contact with a substrate. International Journal of Solids and Structures 44, 1927-1938.

- Lin, Y., and Freund, L.B., 2008. Optimum size of a molecular bond cluster in adhesion. Physical Review E 78, 021909.

- Lin, Y., Inamdar, M., and Freund, L.B., 2008. The competition between Brownian motion and adhesion in soft materials. Journal of the Mechanics and Physics of Solids 56, 241-250.

- Marcy, Y., Prost, J., Carlier, M.-F., and Sykes, C., 2004. Forces generated during actin-based propulsion: A direct measurement by micromanipulation. Proceedings of the National Academy of Sciences 101, 5992-5997.

- McGrath, J.L., Eungdamrong, N.J., Fisher, C.I., Peng, F., Mahadevan, L., Mitchison, T.J., and Kuo, S.C., 2003. The force-velocity relationship for the actin-based motility of Listeria monocytogenes. Current Biology 13, 329-332. 
- Mogilner, A., and Edelstein-Keshet, L., 2002. Regulation of actin dynamics in rapid moving cells: a quantitative analysis. Biophysical Journal 83, 1237-1258.

- Mogilner, A., and Oster, G., 1996. Cell motility driven by actin polymerization. Biophysical Journal 71, 3030-3045.

- Mogilner, A., and Oster, G., 2003. Force generation by actin polymerization II: the elastic ratchet and tethered filaments. Biophysical Journal 84, 1591-1605.

- Munevar, S., Wang, Y.-L., and Dembo, M., 2001. Distinct roles of frontal and rear cell-substrate adhesions in fibroblast migration. Molecular Biology of the Cell 12, 3947-3954.

- Novak, I.L., Slepchenko, B.M., Mogilner, A., and Loew, L.M., 2004. Cooperativity between cell contractility and adhesion. Physical Review Letters 93, 268109.

- Palecek, S.P., Loftus, J.C., Ginsberg, M.H., Lauffenburger, D.A., and Horwitz, A.F., 1997. Integrin-ligand binding properties govern cell migration speed through cell-substratum adhesiveness. Nature 385, 537540 .

- Pollard, T., 1986. Rate constants for the reactions of ATP- and ADPactin with the ends of actin filaments. The Journal of Cell Biology 103, 2747-2754.

- Preston, T.M., King, C.A., and Hyams, J.S., 1990. The Cytoskeleton and Cell Motility. Blackie, Glasgow.

- Rentsch, P.S., and Keller, H., 2000. Suction pressure can induce uncoupling of the plasma membrane from cortical actin. European Journal 
of Cell Biology 79, 975-981.

- Small, J.V., 1994. Lamellipodia architecture: actin filament turnover and the lateral flow of actin filaments during motility. Seminars in Cell Biology 5, 157-163.

- Svitkina, T.M., Verkhovsky, A.B., McQuade, K.M., and Borisy, G.G., 1997. Analysis of the actin-myosin II system in fish epidermal keratocytes: mechanism of cell body translocation. The Journal of Cell Biology 139, 397-415.

- Tan, J.L., Tien, J., Pirone, D.M., Gray, D.S., Bhadriraju, K., and Chen, C.S., 2003. Cells lying on a bed of microneedles: An approach to isolate mechanical force. Proceedings of the National Academy of Sciences 100, 1484-1489.

- Theriot, J.A., and Mitchison, T.J., 1991. Actin microfilament dynamics in locomoting cells. Nature 352, 126-131.

- Ware, M.F., Wells, A., and Lauffenburger, D.A., 1998. Epidermal growth factor alters fibroblast migration speed and directional persistence reciprocally and in a matrix-dependent manner. Journal of Cell Science 111, 2423-2432.

- Wei, Y., 2008. Physical interpretation of the maximum receptor-ligand bond spacing to ensure cell adhesion in ligand-coated Substrates. Langmuir 24, 5644-5646.

- Zaidel-Bar, R., Cohen, M., Addadi, L., and Geiger, B., 2004. Molecular environment of integrins. Biochemical Society Transactions 32, 416420.

- Zamir, E., and Geiger, B., 2001. Components of cell-matrix adhesions. 
Journal of Cell Science 114, 3577-3579. 